\title{
LES POSTES DE JOURNALISTES-MANAGERS, UN ENJEU PROBLÉMATIQUE DE LA CARRIÈRE DES JOURNALISTES
}

\author{
Matthieu LARDEAU ${ }^{1}$
}

\begin{abstract}
Dans cet article, nous nous intéressons à une étape particulière de la carrière des journalistes, la promotion à un poste d'encadrement et à une catégorie particulière de journalistes, les journalistes-managers, c'est-àdire les journalistes qui occupent également des fonctions de management au sein de leur rédaction. Ces postes de management intermédiaire jouent un rôle prépondérant dans le bon fonctionnement des rédactions. À l'appui de l'étude des trajectoires et de l'observation du travail de journalistes-managers de la presse quotidienne nationale, nous montrons que cette dimension particulière de la carrière de journaliste - devenir journaliste-manager - s'inscrit dans une forme de promotion professionnelle. Mais, paradoxalement, ces postes ne comblent pas particulièrement leurs titulaires ou n'attirent pas les candidats les mieux qualifiés. Les directions des journaux, qui nomment les titulaires de ces postes, et les journalistes, qui occupent ces postes, ont des approches divergentes du rôle et de la fonction de journaliste-manager. Ainsi, la définition de ces postes et fonctions, et en particulier les attentes en matière de management d'équipe, ne fait pas l'objet d'une attention particulière de la part des directions de journaux, ce qui démotive et frustre de très nombreux journalistes-managers.
\end{abstract}

1 Matthieu Lardeau est maitre de conférences en sciences de gestion, laboratoire CleRMa/Clermont Recherche Management (EA 3849), Université Clermont Auvergne.

Recherches en communication, $\mathrm{n}^{\circ} 43$ - Article publié le 13/08/2020 
La continuité ou la discontinuité des carrières professionnelles se pose, pour certains journalistes, quand il s'agit d'envisager l'exercice d'un premier poste formel d'encadrement au sein d'une rédaction. Ces postes ne comptent pas en général parmi les plus recherchés par les journalistes alors même qu'ils constituent des rouages essentiels du bon fonctionnement d'une rédaction, d'un média d'information et même du travail journalistique.

Si l'objet gestion ou management appliqué au journalisme reste bien souvent un point aveugle de la recherche, il mérite d'être mobilisé aujourd'hui tant les médias d'information, et singulièrement la presse quotidienne de hard news, sont confrontés à un profond changement institutionnel marqué, notamment, par les prémices de l'introduction de politiques de marketisation, de managérialisation ou de financiarisation. Dans cette configuration, on peut faire l'hypothèse que ces journaux, entrés dans un processus plus ou moins avancé de rationalisation managériale, sont plus enclins à mettre en place des dispositifs de gestion attentive des carrières des journalistes passant par des postes de management, en particulier dans la détection des potentiels, mais aussi à placer les titulaires de ces postes dans les meilleures conditions pour qu'ils mettent en œuvre ces politiques managériales. Nous nommons ici journalistes-managers ces journalistes qui exercent des fonctions managériales ou d'encadrement au sein des rédactions, en parallèle de leurs fonctions éditoriales premières. Ils concernent les titulaires des postes de direction éditoriale de rédactions qui exercent également des fonctions de management (directeur de rédaction, directeur de rédaction adjoint, rédacteur en chef, rédacteur en chef adjoint, secrétaire général de rédaction) ainsi que les titulaires des postes d'encadrement intermédiaire (chef de service et chef de service adjoint).

Cet article repose fondamentalement sur une recherche de terrain menée dans plusieurs rédactions de journaux français, en particulier $L a$ Croix, 20 Minutes et Le Parisien-Aujourd'hui en France (qui constituent notre corpus primaire), entre 2006 et 2011. Nous y avons recueilli des données primaires, en combinant principalement enquêtes ethnographiques, observations participantes et entretiens semi-directifs avec les journalistes-managers et des journalistes non-managers. Les directions de ces trois quotidiens ont accepté notre présence quotidienne au sein de leur rédaction : deux semaines en octobre 2007 (suivies de retours réguliers durant plusieurs mois pour suivre l'évolution de la vie de la rédaction et y mener les entretiens) à La Croix; quatre semaines en juin 2008 (suivies de retours réguliers durant plusieurs mois pour 
suivre l'évolution de la vie de la rédaction et y mener des entretiens restants) à 20 Minutes et cinq mois, d'octobre 2008 à février 2009, au Parisien-Aujourd'hui en France.

Ces périodes d'observation nous ont permis d'agir très librement, de nous intégrer dans les différents services de chaque rédaction, d'assister aux réunions de services, conférences de rédaction quotidiennes et conférences de programmation ou de débriefing hebdomadaires ainsi qu'à certaines autres réunions de direction sur des enjeux éditoriaux, commerciaux ou marketing. Notre priorité consistait à observer et prendre en notes les actions et décisions des journalistes-managers relevant de leurs fonctions éditoriale et managériale. En adoptant la technique du « shadowing ", nous suivions ainsi un journaliste-manager durant plusieurs jours, dès son arrivée à la rédaction jusqu'à son départ en fin de journée. Par la suite, des entretiens individuels semi-directifs en face à face ont été menés avec les journalistes-managers observés, portant sur leur parcours universitaire et professionnel et sur leur approche du management, en leur demandant notamment d'interpréter les observations faites quelques heures ou jours plus tôt de leurs principales décisions. En l'absence d'éléments biographiques suffisamment précis de chaque membre des rédactions avant l'abord des terrains, il était impossible de constituer un échantillon représentatif des journalistes-managers à interroger; nous avons donc cherché à les observer et à les interroger de manière exhaustive, objectif atteint à quelques unités près. Ainsi, 116 entretiens ont été menés au sein de ces trois journaux, dont 90 avec des journalistes (69 journalistes-managers et 21 journalistes sans responsabilités managériales) : 25 à La Croix, 18 à 20 Minutes et 47 au Parisien-Aujourd'hui en France.

Le choix de l'échantillon des journaux repose donc sur deux critères principaux : la recherche de quelques cas représentatifs du secteur étudié et des raisons d'opportunité (l'acceptation de notre présence par les dirigeants des journaux). Les trois journaux qui constituent le corpus principal de notre étude partagent les deux caractéristiques majeures recherchées : ils relèvent du segment de la presse quotidienne nationale d'information générale (print et Internet) et ils appartiennent à trois groupes de presse différents avec un actionnariat stable et peu diversifié. Ces trois journaux répondent également aux particularités recherchées : ils ont été créés à trois périodes différentes et cruciales de l'histoire de la presse et du journalisme français (La Croix en 1883 : débuts de la professionnalisation du journalisme français, Le Parisien en 1944 : Libération et 20 Minutes en 2002 : arrivée des quotidiens 
gratuits), deux sont payants et le troisième est gratuit et ils reposent sur des logiques de création (idéologiques, économiques et politiques, commerciales) différentes. Mais surtout, ces trois journaux sont touchés, à des degrés divers, par le changement institutionnel qui se dessine alors sur ce marché : un journal se trouve volontairement très en retrait de la managérialisation ( $L a$ Croix), un autre s'y est engagé mais semble revenir quelque peu en arrière (Le Parisien-Aujourd'hui en France), un troisième au modèle économique qui repose sur une forte logique marketing et managériale (20 Minutes).

Cet article contribue à la littérature sur le journalisme en complétant notamment des études des trajectoires des journalistes (Devillard et al., 2001 ; Leteinturier, 2002, 2012, 2014, 2015, 2016) par une analyse pionnière des postes de journalistes managers, dans la presse française. Nos résultats montrent que, pour la majorité des journalistes et journalistes-managers observés et interrogés, les postes de management au sein de leurs rédactions respectives ne sont pas épanouissants mais restent avant tout synonymes de promotion professionnelle, de continuité logique de la carrière ; nombre des titulaires de ces fonctions en délaissent la dimension managériale pour se concentrer sur les fonctions éditoriale et journalistique.

Dans une première partie, nous résumons le contexte institutionnel dans lequel s'inscrit la presse de hard news depuis deux décennies, à savoir une pré-institutionnalisation d'une logique managériale, supposée favoriser l'émergence du rôle et de la fonction de journalistemanager. Puis, nous montrons que les postes de journalistes-managers n'attirent pas toujours les profils de candidats attendus. Ensuite, nous identifions que les entreprises de presse, qui déclarent vouloir introduire ou développer la logique managériale, ne créent paradoxalement pas les conditions nécessaires pour que ces postes de journalistes-managers attirent les profils idoines. Enfin, nous soulignons que nombre de titulaires de ces postes expriment leur frustration à l'égard des injonctions paradoxales qui caractérisent l'exercice de leurs fonctions hybrides.

\section{Les journalistes-managers au cour du changement institutionnel}

Cette recherche s'inscrit dans la théorie néo-institutionnelle qui permet de mettre en évidence les différentes logiques institutionnelles ${ }^{2}$

2 Les logiques institutionnelles sont définies comme des schémas historiques et sociaux de pratiques, de croyances, de valeurs et de règles par lesquels les individus 
à l'œuvre dans les marchés de la presse et du journalisme de hard news. Les marchés des médias d'information relèvent d'une configuration de double-sided market (Schudson, 1978) dont le contenu est défini par deux composantes : le contenu éditorial ou rédactionnel (l'information) et le contenu commercial (la publicité). Ces deux contenus relèvent de deux dimensions distinctes et gérées par des professionnels spécifiques - les journalistes et les gestionnaires - et selon des valeurs et principes propres. Les modèles économiques des médias d'information - et singulièrement de la presse de hard news - sont depuis l'origine régis par deux logiques institutionnelles constitutives des tensions de la réalité journalistique (Watine, 2002 ; Raviola, 2017), mais complémentaires : la logique éditoriale et la logique commerciale. La logique éditoriale est portée par les journalistes qui promeuvent et défendent les valeurs professionnelles (du journalisme) tandis que la logique commerciale consiste à développer les revenus tirés du contenu publicitaire et plus largement de la gestion du média qui est aussi une entreprise (Lardeau, 2011). Lorsque ces médias opèrent dans une situation financière assurant une rentabilité significative, les deux logiques entrent rarement en conflit : la logique éditoriale reste la logique qui domine le fonctionnement et la vie des médias d'information. Toutefois, lorsque ces entreprises se trouvent dans une situation de déficit financier structurel, les tenants de la logique commerciale cherchent à ce que leurs principes et valeurs dominent la gestion quotidienne des médias pour en faire des entreprises rentables, cette logique entrant le plus souvent en conflit avec la logique éditoriale.

Caractérisée par un déficit financier structurel (Le Floch \& Sonnac, 2013), la presse de hard news en France connaît un changement institutionnel profond qui se traduit par la mise en œuvre - ou à tout le moins la recherche de mise en œuvre - de politiques de financiarisation (Almiron, 2010), de marketisation (McManus, 1994) et de managérialisation (Underwood, 1993) au sein des journaux de hard news et de leurs rédactions. Ces politiques initiées par les directions générales de journaux - et parfois de nouveaux actionnaires - se présentent comme des processus qui consistent à introduire, au sein des rédactions, les principes, discours et pratiques de rationalisation managériale. L'objectif

produisent et reproduisent leur subsistance matérielle, organisent le temps et l'espace et donnent un sens à la réalité sociale (Thornton \& Ocasio, 1999. Institutional logics and the historical contingency of power in organizations: Executive succession in the higher education publishing industry, 1958-1990. American Journal of Sociology 105(3), 804). 
consiste à modifier fondamentalement les pratiques de gestion des journaux et des rédactions pour les rationaliser, c'est-à-dire les faire tendre vers une plus grande productivité, à la fois en termes de rendement et de profit. À terme, il s'agit d'assurer une gestion rationalisée et rentable de ces entreprises. La mise en œuvre de ces processus repose sur les individus qui, tout en restant journalistes, sont donc, plus ou moins, investis également de tâches managériales : les journalistes-managers.

La littérature académique française néglige généralement la dimension managériale du journalisme et plus précisément l'étude des journalistes-managers (pour quelques exceptions : Padioleau, 1985 ; Charon, 1991 ; Lardeau, 2011). Ce concept est pourtant documenté par la littérature anglo-saxonne depuis plusieurs décennies à travers l'étude du Publisher (Padioleau, 1985), de l'Editor as manager (Bennett, 1985), des newsroom middle managers (Giles, 1987 ; Fink, 1988 ; Lavine \& Wackman, 1988 ; Underwood, 1993 ; Allen, 1995 ; Gade, 2002 ; Müllern, 2006 ; Raviola, 2017), etc. Les seules études d'envergure sur le cas français consacrées à la description et à l'analyse des populations et profils de journalistes font référence à la notion de « journaliste-cadre » (Devillard et al., 2001, p. 46) ou de « cadre dirigeant journaliste » qui désigne les " journalistes ayant atteint des positions d'encadrement, depuis celle de chef de rubrique jusqu'à celle de directeur en passant par celle de rédacteur en chef » (Leteinturier, 2002, p. 124, note 10).

\section{Des postes d'encadrement choisis pour leur dimension éditoriale au détriment du management}

Nous avons pu observer puis interroger la quasi-totalité des directeurs de rédaction, rédacteurs en chef, chefs de service et chefs de service adjoints des trois journaux qui constituent notre corpus primaire - notre corpus secondaire étant composé d'autres journaux ou organisations professionnelles de médias au sein desquels nous n'avons pas mené d'observations participantes mais uniquement des entretiens individuels. Il ressort très nettement que l'immense majorité de ces journalistes-managers, par-delà les conditions spécifiques de chaque rédaction, n'éprouvent aucun attrait fondamental pour les fonctions managériales, au début comme en milieu de carrière. Ce constat vaut tant pour les fonctions de middle management ou management intermédiaire (chefs de service et adjoints) que celles de top management (rédacteur en chef et directeur de rédaction et adjoints). 
L'attrait majeur pour les postes d'encadrement réside dans l'exercice (pour les chefs de service) ou l'accroissement (pour les rédacteurs en chef) de responsabilités éditoriales permettant ainsi d'agir directement sur le contenu éditorial et rédactionnel de son service et du journal et répond au souhait d'accepter une promotion vécue comme une reconnaissance professionnelle. L'immense majorité des journalistes expliquent leur faible attirance pour la dimension d'encadrement, de management, de gestion d'équipe de ces postes (en particulier les premiers postes d'encadrement : chef de service adjoint puis chef de service) en invoquant l'argument de l'identité professionnelle de journaliste. Cette dernière, toujours en questionnement (Ruellan, 1993 ; Lemieux, 2000), porte les journalistes à s'intéresser prioritairement à la recherche et à l'analyse de l'information plutôt qu'aux questions de gestion, de management. «J'ai choisi d'être journaliste pour recueillir de l'info, être sur le terrain presque chaque jour, pour rencontrer de nouvelles personnes et être dans un état de surprise vis-à-vis de l'actu. Tout cela s'oppose aux fonctions d'encadrants qui obligent à s'éloigner du terrain pour gérer les conflits et les ego des confrères, les plannings, relire de la copie, être dépendant de la hiérarchie! "», explique un chef de service à La Croix dont les propos représentent ceux exprimés par la quasi-totalité de ses confrères lors de notre étude. Cela vaut encore plus pour l'adjoint que le chef de service : ce dernier, selon sa personnalité et le degré de respect qu'il porte à ses adjoints, peut se " décharger » sur ces derniers des tâches les plus routinières (relecture) et les moins gratifiantes ou qui les exposent à des conflits de personnes (gestion au quotidien des conflits avec les journalistes, planning, etc.)

De même, les postes de rédacteur en chef, en nombre plus restreint, attirent tout autant les candidatures. Les nominations y sont encore plus contrôlées et relèvent d'une relation de don/contre-don. En règle générale, on y accède après être passé par des fonctions de chef de service et/ou adjoint et avoir fait montre d'une grande loyauté à l'égard de la rédaction. À La Croix, l'un des rédacteurs en chef déclare : " quand la proposition m'a été faite, j'ai d'emblée refusé à cause de la masse de travail à abattre (aussi lourde mais différente de celle d'un chef

3 Tous les verbatim cités dans cet article sont issus des terrains de recherche présentés. Nous les avons sélectionnés en respectant un double critère : (1) être très expressifs, c'est-à-dire rendre compte, de la manière la plus claire et la plus compréhensible, de l'interprétation donnée par notre répondant et (2) être représentatifs du discours majoritaire entendu lors de nos enquêtes de terrain. 
de service) mais surtout qu'une grande partie du travail au quotidien consiste à relire les pages! Ce qui m'a décidé finalement, c'est la perspective de participer à un vrai travail d'équipe dans la définition et la traduction de la politique éditoriale du titre : aujourd'hui, je ne regrette pas car ce travail d'équipe existe et nous pousse à rechercher l'excellence éditoriale... et atteindre des objectifs de vente ! " Dans notre corpus, nous n'avons pas observé de cas de refus d'une telle proposition qui traduit une promotion éminemment valorisante et qui s'apparente à une forme d'accomplissement professionnel dans une carrière.

Néanmoins, tant à La Croix qu'au Parisien, les secrétaires généraux de rédaction font état d'une situation qui peut paraître paradoxale. Les vacances de postes de chef de service et chef de service adjoint - ce dernier poste permet d'assurer l'intérim à la tête du service en l'absence $\mathrm{du}$ titulaire et par la suite prétendre à lui succéder - appellent un nombre toujours élevé de candidatures mais "l'écrasante majorité des candidatures ne répond pas à nos attentes. Ces candidats, qui peuvent être de bons journalistes, s'imaginent ipso facto bons managers alors que ce n'est pas souvent le cas. De simples questions montrent déjà l'absence de connaissances minimales et de véritable appétence pour le côté managérial de la fonction de chef de service, voire de rédacteur en chef », précise un secrétaire général de rédaction.

\section{Une fonction et un rôle mal définis : recruter des leaders, des managers ou des animateurs?}

Les groupes de presse quotidienne expriment, nolens volens, la volonté de bien définir les postes d'encadrement au sein de leurs rédactions, en particulier leur dimension managériale. Le secrétaire général de la rédaction du Parisien explique ainsi sa conception du profil et des responsabilités des chefs de service au sein de sa rédaction : "nous faisons en sorte que nos encadrants soient de vrais journalistesmanagers, et donc un peu plus managers qu'habituellement. Mais nous partons de très loin, même chez nous, car la culture de la rédaction est à $100 \%$ concentrée sur le contenu, le journalisme. Depuis toujours, les chefs de service développent une expertise éditoriale et journalistique leur donnant l'autorité naturelle pour diriger d'autres journalistes. Dans notre projet, ils doivent conserver tout cela et ajouter des compétences en management de service, d'équipe pour être en mesure de gérer rationnellement leurs services et équipes, de 
manière plus efficace, en tenant compte de la dimension prévisionnelle de la fonction, notamment en gérant leur budget. Évidemment, il est hors de question d'aller jusqu'à mettre en place une organisation très rationalisée comme dans d'autres entreprises en dehors de la presse. ”

Nous constatons toutefois que les différentes catégories d'acteurs au sein des rédactions disposent d'approches divergentes de ce que recouvrent la fonction et le rôle de journaliste-manager, au-delà des quelques propos génériques communs.

\subsection{Quelles qualités sont recherchées chez les journalistes- managers?}

Les définitions et perceptions des qualités requises pour occuper ces fonctions sont généralement divergentes. Les seules convergences s'observent sur l'identification très générique des grandes qualités requises : premièrement, faire état d'une carrière de journaliste qui doit montrer que celui-ci maîtrise toutes les qualités professionnelles attendues chez un journaliste (sens de l'actualité et réactivité propre à la presse quotidienne, qualités rédactionnelles, maîtrise de la chaîne de production éditoriale, passion du métier et engagement personnel). Cette qualité première attendue énonce que le journaliste-manager doit d'abord être reconnu comme un (très) bon journaliste. La deuxième qualité relève de la maîtrise de la ligne éditoriale globale du journal et le sens de la hiérarchie de l'information conséquente. Ces deux premières qualités recherchées montrent que la légitimité du journaliste-manager s'inscrit avant tout dans son identité professionnelle de journaliste et non de manager. Enfin, il faut avoir démontré - ou a minima présenter - le potentiel d'être « chef » ou « manager » selon le niveau de responsabilités d'encadrement (service ou rédaction), c'est-à-dire au minimum disposer des aptitudes pour gérer une équipe et un service (ou une rédaction et le journal).

L'asymétrie s'observe quand il s'agit de définir ce que recouvre la dimension de manager mais aussi de pondérer les trois qualités précitées. Les directions des journaux souhaitent que la fonction managériale prenne de plus en plus d'ampleur alors que les journalistes-managers considèrent que la dimension managériale de leur fonction doit rester secondaire, voire accessoire au regard de la dimension éditoriale et journalistique dominante. Ainsi, les rédacteurs en chef s'accordent pour dresser les qualités à réunir pour exercer leurs fonctions : grande légitimité journalistique, curiosité et sens de l'actualité pour être capable 
de « jongler » entre toutes les rubriques d'un journal et maîtriser les conditions du possible de cette fonction, c'est-à-dire savoir apprécier la marge de manœuvre dont on dispose et savoir ne pas empiéter sur le territoire de la direction de la rédaction... Ces qualités ressortissent de la logique éditoriale/journalistique : peu évoquent en effet des qualités managériales, ce qui s'explique par le fait de ne pas avoir à diriger directement une équipe. Toutefois, leur hiérarchie attend d'eux également qu'ils exercent pleinement la dimension managériale de leur fonction, en se comportant comme des leaders sur l'ensemble de la rédaction. Il s'agit alors de jouer le rôle de médiateur des idées et pratiques de rationalisation managériale.

Les directions des trois journaux observés, en particulier au Parisien (et à un degré moindre à La Croix où prédomine la logique éditoriale et à 20 Minutes dont la rédaction, plus réduite numériquement, présente moins de postes à responsabilités managériales chez leurs encadrants), tendent à orienter la fonction de manager dans le sens de leader, de leadership alors que les journalistes-managers, en particulier les chefs de service, développent une approche minimale, celle d'animateur d'équipe ou de service. Ainsi, tous les chefs de service observés puis interrogés, dans les trois journaux de notre corpus, circonscrivent volontairement l'approche de la dimension managériale de leur fonction au fait de rester tout au plus un animateur et non un leader : " ce qui me plaît le plus dans cette fonction, explique un chef de service à La Croix, c'est d'animer mon service, c'est-à-dire jour après jour faire vivre le débat, remuer les idées, faire vivre l'intelligence produite par mon équipe pour proposer le meilleur contenu éditorial de mes pages. Trouver l'équilibre entre ces personnalités et gérer leurs états d'âme, c'est déjà suffisant! Je fais tout cela à l'intuition et non de manière rationnelle. Pour moi, c'est cela être chef de service, pas plus. Le reste des questions managériales (le reporting, les chiffres, le budget, etc.) ne m'intéresse pas et je pense qu'elles n'ont pas leur place dans notre journal. »

Cette approche minimaliste de la fonction de chef de service n'est pas propre à un journal encore dominé par la logique institutionnelle éditoriale. Les confrères de 20 Minutes et du Parisien-Aujourd'hui en France - journaux plus engagés dans le processus de managérialisation - ne disent pas autre chose. "À la limite, je ne serais pas contre m'investir un peu plus dans le management de mon service mais encore faut-il en avoir le temps et parfois les moyens ", explique ce chef de service à La Croix et l'un de ses confrères du Parisien de préciser : «on 
doit produire toujours plus de contenu, toujours plus vite et comme nous cherchons à maintenir le plus possible de pages chaudes pour coller à l'actu, eh bien nous n'avons pas matériellement le temps nécessaire de nous investir dans le management et la gestion des ressources humaines qui réclament du recul, du temps et de l'investissement car ce n'est pas le cour de notre métier!"»

\subsection{La définition lacunaire des fonctions de chef de service et chef de service adjoint}

Ce décalage de perception, entre journalistes et directions de rédaction, de ce que recouvrent les postes de journalistes-managers - surtout ceux de chefs de service - ressortit également de l'absence de définition précise de ces postes, voulue par les directions de journaux. L'absence d'affichage et d'objectivation des critères recherchés permet ainsi à la direction de conserver la mainmise sur les processus de recrutement et de promotion interne, en se préservant l'opportunité de mobiliser des critères ad hoc lors de chaque promotion. Les directeurs de rédactions et directeurs des ressources humaines interrogés sur les définitions de postes sont restés peu loquaces, ce que regrette ce journaliste-manager à La Croix: "la DRH refuse de fournir une définition précise des postes d'encadrement et je suis persuadé que la DRH elle-même ne connaît pas les critères et qualités requis pour ces postes car elle est très éloignée de nos réalités, de nos métiers!"

Nous observons enfin une asymétrie dans l'approche de la fonction très particulière de chef de service adjoint. Ces postes constituent une première expérience du management d'équipe : cela permet aux journalistes de « se tester » et de pouvoir, éventuellement, "juger sur pièces » leurs compétences et appétence pour les fonctions d'encadrement. En retour, les aux directions des rédactions ont l'opportunité de détecter les futurs chefs de service, tout en précisant que, dans la majorité des cas, cette détection ne répond à aucune politique rationnalisée et objectivée tant de la part des directions de rédaction que de celle des ressources humaines mais relève plutôt d'évaluations ad hoc, empiriques et intuitives. Les représentations divergent également sur la définition du périmètre de la fonction (quelles sont les tâches et missions précises, en termes de management et de gestion d'équipe, que la direction attend de ses journalistes-managers). Cette définition dépend in fine largement de la personnalité du chef de service et de son souhait de choisir, en accord avec sa direction, un adjoint qui lui convienne. Dans une même 
rédaction, nous avons comparé deux services équivalents (productivité quotidienne en termes de papiers, taille du service, place et rôle dans la stratégie éditoriale du journal, etc.) dont les chefs développent des pratiques opposées à propos de la gestion de leurs adjoints. Quand l'un s'attache à beaucoup déléguer (et pas seulement les tâches les moins nobles comme par le suivi du planning, la gestion des brèves, la relecture, etc.) avec pour objectif de se constituer une équipe d'adjoints performante et travailler dans la cohésion, son voisin confie essentiellement à son adjoint les tâches qui l'intéressent peu ou pas. Comme le reconnaît un journaliste de La Croix, "la fonction d'adjoint de chef de service est probablement la plus ingrate dans une rédaction. C'est la moins reconnue, la plus routinière, celle qui demande une grande organisation, une résistance au stress quand le chef de service se comporte en autocrate ou est un mauvais chef et c'est la seule fonction d'encadrement qui, paradoxalement, ne fonctionne pas à l'ego : il faut être au service du chef et des collègues, sans "broncher". Il faut beaucoup "prendre sur soi" comme on dit ».

\subsection{Des journalistes-managers privés de moyens et d'outils de management}

Une autre question illustre le décalage de perception entre directions et journalistes-managers : celle des moyens alloués à ces derniers, en particulier les chefs de service, pour remplir leur tâche de manager d'équipe. Les discours des équipes dirigeantes des trois journaux étudiés convergent pour annoncer mettre en place des politiques managériales destinées à donner à leurs journalistes-managers les moyens de développer leurs capacités de leadership. Toutefois, nous avons constaté que la réalité de l'exercice des fonctions des journalistesmanagers, en particulier les chefs de service, reste éloignée des discours. Un indicateur parmi d'autres illustre ce constat : l'étendue et la qualité de l'information diffusée à propos des chiffres et données relatifs à l'économie et la gestion des journaux. Toutes les directions des journaux étudiés, à des degrés divers, requièrent de leurs chefs de service qu'ils s'impliquent davantage dans la connaissance et la compréhension des grands équilibres commerciaux de leur entreprise, ce qui implique notamment de disposer de toutes les données marketing, commerciales, publicitaires, voire stratégiques. Nous avons pu constater néanmoins que les directions de journaux ne leur fournissent pas les moyens effectifs pour atteindre cet objectif. La diffusion de l'infor- 
mation commerciale et marketing reste bien souvent peu claire ou peu lisible pour des non-spécialistes et elle est transmise par des membres du service commercial ou marketing dont les discours et les présentations ne convainquent que rarement les journalistes-managers. À la sortie des réunions dans lesquelles ces éléments sont diffusés (et auxquelles nous avions assisté), les chefs de service nous faisaient part de leur déception de ne pas pouvoir tirer de véritables enseignements de ces présentations, faute de temps et d'outils de connaissance pour approfondir la réflexion. Ils se montraient peu convaincus par les explications fournies en fin de conférence de rédaction par le représentant du service commercial.

À titre d'illustration, un chef de service - doté d'une longue expérience entrepreneuriale (durant son parcours professionnel, il a créé puis dirigé une petite agence de presse, développant ainsi des compétences en gestion, marketing et management) - analyse ainsi la situation dans son journal : " on nous demande d'être des managers, des leaders parfois mais on ne nous donne pas tous les moyens ni même les prérogatives indispensables pour exercer ce type de mission. Par exemple, on nous demande de faire prendre conscience à nos journalistes de l'importance de la partie commerciale, de l'apport possible du marketing, de prendre conscience de l'équilibre économique et financier du journal. Moi, je ne suis pas contre, bien au contraire, sans que cela dépasse la ligne jaune. Mais à chaque fois que je demande des chiffres plus précis sur les ventes, les abonnements, les résultats des études marketing pour en diffuser certains éléments auprès de mon service, eh bien la direction de la rédaction ne répond pas ou botte en touche : nous devons nous contenter des quelques éléments diffusés via l'Intranet mais ceux-ci sont insuffisants pour comprendre la situation du journal. La plupart des données sont considérées comme stratégiques et ne sont divulguées qu'à la direction de la rédaction et parfois à la rédaction en chef : les chefs de service n'y ont pas accès ! Je ressens cela comme une infantilisation inacceptable et pas loyale! On nous fait comprendre que nous ne serions pas assez intelligents ou formés à la gestion pour prendre la juste mesure de ces données ! » Un confrère d'un autre journal ajoute : "la question des budgets des services est également révélatrice. On nous donne l'illusion de disposer d'un budget à gérer pour notre service mais on connaît très mal les critères et règles de définition de leur périmètre et de la répartition entre services. Là encore, cela manque de transparence de la part de la direction et du secrétariat général et les discours ne sont pas en adéquation avec les actes! » 


\section{Conclusion. Des journalistes-managers frustrés et lassés des injonctions paradoxales}

Les journalistes-managers vivent une situation « de frustration » que résume un chef de service adjoint au Parisien : "nous vivons constamment au milieu d'injonctions paradoxales. D'un côté, on nous demande d'être plus responsables, de devenir des leaders au sein de nos services pour les gérer plus efficacement et de l'autre, on crée des conditions contraires à cette responsabilisation. Par exemple, nous devons de préserver l'actu chaude, la recherche de scoops mais, dans la réalité, on nous demande de boucler toujours plus tôt pour raisons économiques. Ou alors on demande au chef de service d'être une force de propositions au sein de la rédaction, pas seulement sur le plan éditorial mais aussi sur le plan de l'organisation et du management pour améliorer l'efficacité du fonctionnement de la rédaction mais finalement. On se rend compte que les journalistes et encadrants qui sont récompensés sont les plus obéissants, les plus légitimistes et ceux qui ont "joué le jeu" sont sanctionnés!"

La frustration est d'autant plus grande chez les journalistes-managers ou journalistes qui possèdent les compétences et les connaissances pour interpréter des données marketing et de gestion, même complexes. Ces derniers, néanmoins minoritaires dans notre cohorte, ont acquis les compétences et l'appétence pour les questions de gestion au gré d'une expérience professionnelle antérieure, dans la presse magazine (où les postes de journalistes-managers sont plus développés et plus exigeants quant à la dimension gestion et management) ou en dehors de la presse (par exemple dans des fonctions commerciales). Plus rarement, cette connaissance minimale de ces questions a pour origine une formation universitaire en management des médias : si ces formations (Masters) se sont développées en France depuis une vingtaine d'années, elles n'ont été suivies que par quelques journalistes et journalistes-managers rencontrés. Quant aux diplômes de journalisme délivrés par les écoles de journalisme, ils ne proposent qu'un enseignement en économie et gestion des médias (un cours semestriel obligatoire fait partie des exigences pour que le diplôme soit « reconnu par la profession »). Dans leur grande majorité, les journalistes-managers se disent " frustrés » par l'exercice de leur double fonction. Certains, lassés de ces pratiques " infantilisantes », ne s'impliquent plus autant qu'avant dans leurs tâches managériales ("maintenant, j'assure le service minimum ", explique un chef de service) et voient dans ces pratiques une mesure de 
défiance à leur égard, les interprétant comme une invitation à quitter le journal (ce qu'ont fait certains d'entre eux).

Notre étude met en lumière le décalage entre les discours des trois groupes de presse - invitant leurs employés à innover, à entreprendre en ces temps de turbulences pour la presse - et leurs politiques de gestion des rédactions. Ces dernières trahissent, au pire, une résistance à la responsabilisation managériale effective des journalistes de la part des directions de journaux ; au mieux, une absence de politiques managériales effectives pour détecter et accompagner la carrière de ces journalistes-managers potentiels. En effet, les différentes DRH et directions de rédaction interrogées ne connaissent pas - ou ne cherchent pas à connaître - précisément les compétences en marketing et management de leurs journalistes avant de leur proposer des postes de management. Nous constatons également, à la différence de ce qui se pratique dans de grandes entreprises d'autres secteurs, que les groupes de presse quotidienne ne mettent pas en place de politiques de gestion et d'accompagnement de carrière des futurs managers. De même, les quelques formations en management d'équipe - au Parisien-Aujourd'hui en France par exemple - à destination de leurs middle managers ne sont pas suivies de politiques d'accompagnement qui permettraient de rendre effectives et profitables ces formations et d'entretenir un processus de responsabilisation managériale progressive.

Pour conclure, ces premières constatations invitent à poser un regard plus global sur la presse et les médias d'information qui sont traversés par ce changement institutionnel, pour interroger plus en profondeur le comportement observé des journalistes-managers. Ces derniers semblent rechercher une sorte de ligne de conduite équilibrée qui permette de protéger leur rédaction et le métier de journaliste de l'intrusion exagérée de la logique commerciale (marketing et managériale) promue par leurs direction et actionnaires, tout en mettant en œuvre certains éléments, qu'ils jugent pertinents, de rationalisation des modalités de gestion de leur rédaction. Des travaux postérieurs pourraient par exemple poursuivre l'analyse du rôle central de journaliste-manager dans ces processus de rationalisation gestionnaire pour déterminer si cet acteur tend plutôt à devenir un rouage de la mise en œuvre de ces politiques de rationalisation ou à chercher une voie hybride, médiane, empruntant des éléments de marketing, de management ou de gestion financière pour les intégrer à la logique éditoriale et journalistique. 


\section{Références}

Allen, C. (1995). Priorities of General Managers and News Directors in Anchor Hiring. Journal of Media Economics 8(3), 11-124. Disponible à : https://doi.org/10.1207/ s15327736me0803 3

Almiron, N. (2010). Journalism in Crisis: Corporate Media and Financialization. Cresskill: Hampton Press.

Bennett, D. (1985). Editors as Managers: Their Perceived Need for Specialized Training. Newspaper Research Journal 6(4), 24-36. Disponible à : https://doi. org/10.1177/073953298500600404

Charon, J.-M. (1991). La Presse en France, de 1945 à nos jours. Paris : Seuil/Points.

Devillard, V. et al. (Ed.) (2001). Les Journalistes français à l'aube de l'an 2000. Profils et parcours. Paris : Panthéon-Assas.

Fink, C. (1988). Strategic Newspaper Management. Carbondale: Southern Illinois University Press.

Gade, P. (2002). Managing Change: Editors' Attitudes Toward Integrating Marketing Journalism. Newspaper Research Journal 23(2-3), 148-152. Disponible à : https:// doi.org/10.1177/073953290202300213

Giles, R. (1987). Newsroom Management: A Guide to Theory and Practice. Indianapolis: Berg.

Lardeau, M. (2011). Changement institutionnel et managérialisation : transformation de la presse quotidienne nationale française et des pratiques professionnelles journalistiques (1944-2010). Thèse pour le doctorat en sciences de gestion (ESSEC).

Lavine, J. \& Wackman, D. (1988). Managing Media Organizations, Effective Leadership of the Media. New York: Longman.

Le Floch, P. \& Sonnac, N. (2013). Économie de la presse à l'ère numérique. Paris : La Découverte.

Lemieux, C. (2000). Mauvaise presse. Une sociologie compréhensive du travail journalistique et de ses critiques. Paris : Métailié.

Leteinturier, C. (2002). Devenir cadre dirigeant journalistes dans les médias français. Une analyse des biographies publiées par la Correspondance de la presse entre juin 1999 et mai 2000. Dans R. Rieffel \& T. Watine (dir.), Les Mutations du journalisme en France et au Québec (pp. 121-158). Paris: Panthéon-Assas.

Leteinturier, C. (2012). Les carrières de journalistes dans les médias locaux et régionaux : étude de deux cohortes de «nouveaux entrants » (1990-1998). Sciences de la Société 84/85, 265-290.

Leteinturier, C. (2014), Devenir journaliste en presse et le rester : un double défi. Dans C. Leteinturier (Ed.), Les Journalistes français et leur environnement : 19902012. Le cas de la presse d'information générale et politique (pp. 13-55). Paris : Panthéon-Assas.

Leteinturier, C. (2015). Construire les carrières des journalistes titulaires de la carte de presse. L'exploitation des dossiers de la CCIJP. Dans C. Leteinturier \& C. Frisque (Eds), Les Espaces professionnels des journalistes. Des corpus quantitatifs aux analyses qualitatives (pp. 173-199). Paris : Panthéon-Assas.

Leteinturier, C. (2016). Continuité/discontinuité des carrières des journalistes français encartés. Étude de deux cohortes de nouveaux titulaires de la carte de presse. Recherches en Communication 43, 27-55. 
McManus, J. (1994). Market-Driven Journalism: Let the Citizen Beware? Thousand Oaks: Sage.

Müllern, T. (2006). Middle Managers' Identity Work in a Media Context. Dans L. Küng (Dir.), Leadership in the Media Industry: Changing Contexts, Emerging Challenges (pp. 29-40). Jönköping: JIBS Research Report Series \#2006-1.

Padioleau, J.-G. (1985). « Le Monde » et le «Washington Post ». Paris : PUF.

Raviola, E. (2017). Meetings Between Frames: Negotiating Worth Between Journalism and Management. European Management Journal 35(6), 737-744. Disponible à : https://www.sciencedirect.com/science/article/abs/pii/S0263237317300890

Ruellan, D. (1993). Le Professionnalisme du flou. Identité et savoir-faire des journalistes français. Grenoble : Presses universitaires de Grenoble.

Schudson, M. (1978). Discovering the News: A Social History of American Newspapers. New York: Basic Books.

Underwood, D. (1993). When MBAs Rule the Newsroom: How the Marketers and Managers are Reshaping Today's Media. NewYork: Columbia University Press.

Watine, T. (2002). Entre logique marchande et logique citoyenne : une tension constitutive de la nouvelle réalité journalistique ? Dans R. Rieffel \& T. Watine (Dir.), Les Mutations du journalisme en France et au Québec. Paris : Panthéon-Assas. 
(c) (i) () $\bigodot_{\text {BY NC ND }}$ Publié sous la licence Creative Common «Attibution - pas d'utilisation Commerciale - Pas de Modification 4.0. International» (CC BY-NC-ND) 